\section{Simulating actuator energy consumption for trajectory optimisation}

2018, Vol. 232(II) 2178-2192

(C) IMechE 2017

Article reuse guidelines:

sagepub.com/journals-permissions DOI: 10.1177/095441001771027|

journals.sagepub.com/home/pig

\author{
Michael Cooper, Craig Lawson and Amir Zare Shahneh
}

(SAGE

\begin{abstract}
This work aims to construct a high-speed simulation tool which is used to quantify the dynamic actuator power consumption of an aircraft in flight, for use within trajectory optimisation packages. The purpose is to evaluate the energy penalties of the flight control actuation system as an aircraft manoeuvre along any arbitrary trajectory. The advantage is that the approximations include major transient properties which previous steady state techniques could not capture. The output can be used to provide feedback to a trajectory optimisation process to help it compute the aircraft level optimality of any given flight path.

The tool features a six degree of freedom dynamic model of an aircraft which is combined with low frequency functional electro-mechanical actuator models in order to estimate the major transient power demands. The actuator models interact with the aircraft using an aerodynamic load estimator which generates load forces on the actuators that vary as a function of flight condition and control surface demands. A total energy control system is applied for longitudinal control and a total heading control system is implemented to manage the lateral motion. The outer loop is closed using a simple waypoint following guidance system with turn anticipation and variable turn radius control. To test the model, a simple trajectory analysis is undertaken which quantifies a heading change executed with four different turn rates. The tool shows that the actuation system requires 12.8 times more electrical energy when performing a $90^{\circ}$ turn with a radius of $400 \mathrm{~m}$ compared to $1000 \mathrm{~m}$. A second test is performed to verify the model's ability to track a longer trajectory under windy conditions.
\end{abstract}

\title{
Keywords
}

Actuator power, trajectory optimisation, simulation, actuation, airframe systems, secondary power

Date received: 9 September 2016; accepted: 24 April 2017

\section{Introduction}

A simulation tool is developed in this paper which quantifies dynamic actuator power consumption during waypoint-following aircraft manoeuvres of a small $3.6 \mathrm{~m}$ wingspan unmanned aerial vehicle (UAV). It improves upon steady state methods for estimating actuator power consumption (e.g. duty cycle approximations) by including the major electrical and mechanical actuator dynamics.

This work contributes to an effort to achieve fast aircraft architecture simulations for trajectory optimisation; capable of modelling complex dynamic multi-system situations. One example is navigating an icing cloud. Depending on the severity of the conditions, the pilot is presented with the decision of flying through the weather system with anti-icing systems engaged or to navigate around or over it. Each path will have its own effect on the energy demands of the engines, environmental control system, ice protection and flight controls.

Many of these scenarios can be simulated with simple point mass models since they are dominantly based on altitude, airspeed and environmental conditions. The same cannot be said for the primary flight control actuation system because the aircraft dynamic motion directly relates to the flight control power consumption. To simulate the transient behaviour of all three primary control effectors, a six degree of freedom (6-DOF) dynamics model is required to generate control surface deflection commands.

This work contributes to the field of aircraft trajectory optimisation, by evolving the standard aircraft point mass performance models to simulate all six degrees of freedom and incorporating actuation system models. When combined with other airframe system, engine and secondary power generation models the fuel burn for thrust and off-takes can be

Cranfield University, Cranfield, Bedfordshire UK

Corresponding author:

Craig Lawson, Cranfield University, College Road, Cranfield MK43 OAL, Bedfordshire, UK.

Email: c.p.lawson@cranfield.ac.uk 
estimated while following dynamic three-dimensional flight trajectories.

The method demonstrates the capability of using modern, high performance desktop computers to simulate complex aircraft and systems interaction. The end result (which is currently lacking accurate engine and electrical generation models) is a tool which allows the 6-DOF flight dynamic simulation of an aircraft, whilst also producing estimates of instantaneous electrical power as the aircraft flies and manoeuvres.

The block diagram of the tool is shown in Figure 1. At the base of the simulation is the aircraft model, which represents the flight dynamics of the vehicle. The aircraft is controlled using a total energy control system with trajectory guidance. In between the control system and the aircraft lies the actuator models; often these are first- or second-order transfer function models, but in this case the motor and ball screw properties of an electromechanical (EMA) actuator are modelled. The load force on the actuators is derived from an aerodynamic load estimator, which takes into account conditions such as altitude, airspeed and control surface geometry in generating estimates.

When supplemented with other energy quantification, emission and air traffic management models, the tool forms part of the wider dynamic performance model shown in Figure 2, which estimates multiple penalties of the aircraft and its planned trajectory. In the authors' previously published work ${ }^{1}$ high-speed

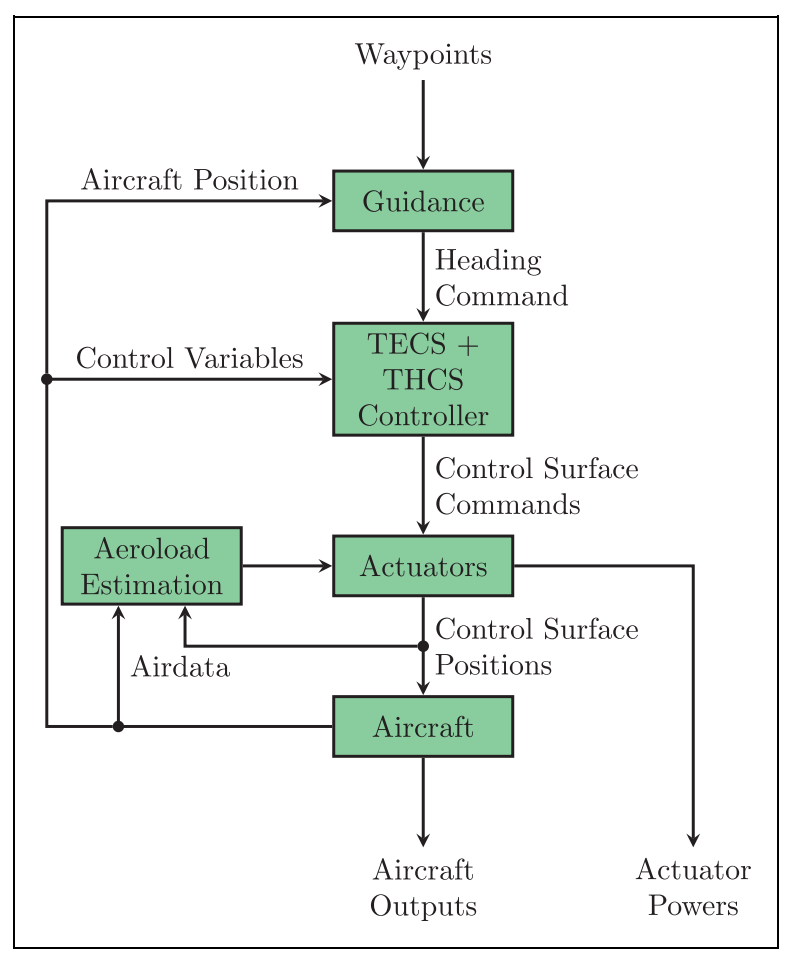

Figure I. Block diagram of the complete aircraft-actuator simulation package. electro-hydrostatic and servohydraulic actuator models are constructed which can replace the EMA unit in this work. This allows a wider range of actuation technologies to be studied, however for the scope of this paper this is not deemed suitable due to the small size of the aircraft.

This paper investigates a single use of the tool; quantifying the energy consumed by the actuators for a series of trajectories. The trajectories are to be simple banked turns between two straight line flight path segments, so that the results are easily verifiable.

Despite high execution speed being a primary goal in the creation of the model, it may still be too slow for online trajectory optimisation problems. Ideally, a high-speed optimiser and point mass aircraft model would be used to generate potential trajectories which are then used as inputs to the 6-DOF with systems model. This tool then serves as a post processing package which 'flies' along the route, producing actuator power consumption data which could not be generated using the point mass model.

\section{Model definition}

\section{Actuator models}

The actuator represented in this work is an EMA type, taken from the authors' previously published work $^{1}$ and simplified for faster simulation. The modelling diagram for the actuator is shown in Figure 3; it consists of a motor driving a load through a gearbox and lead screw combination with total ratio $p$. The equivalent schematic in Figure 4 shows how the gearbox and screw converts the rotational position $\theta_{m}$ from motor $\mathbf{M}$ to translational position $x_{m}$. The relative linear positions of the lead screw nut and load mass define the force developed by the material stiffness and applied to the load. The EMA friction is represented through a viscous damper referred to the actuator case, although additional nonlinear modes can be added as well. Importantly, the force applied to the actuator by the load and friction is reflected back through the gearing to the motor, allowing the power consumption and effects of loading to be studied.

The model is reduced from the referenced document (which was based on a 2-DOF EMA model provided by $\mathrm{Du}$ et $\mathrm{al}^{2}{ }^{2}$ ) to only include a single DOF. This means the model does not provide accurate simulation of the high frequency response of the actuator, since the spring-damper dynamics of the lead screw material are ignored. Consequently the model is only valid when the frequency of excitation is below the actuators' internal resonances. Since the purpose of the tool is a functional dynamic performance model rather than for controller design it is appropriate for the task. The models' predictions are not intended to precisely match experimental hardware dynamics, because their purpose is to produce 


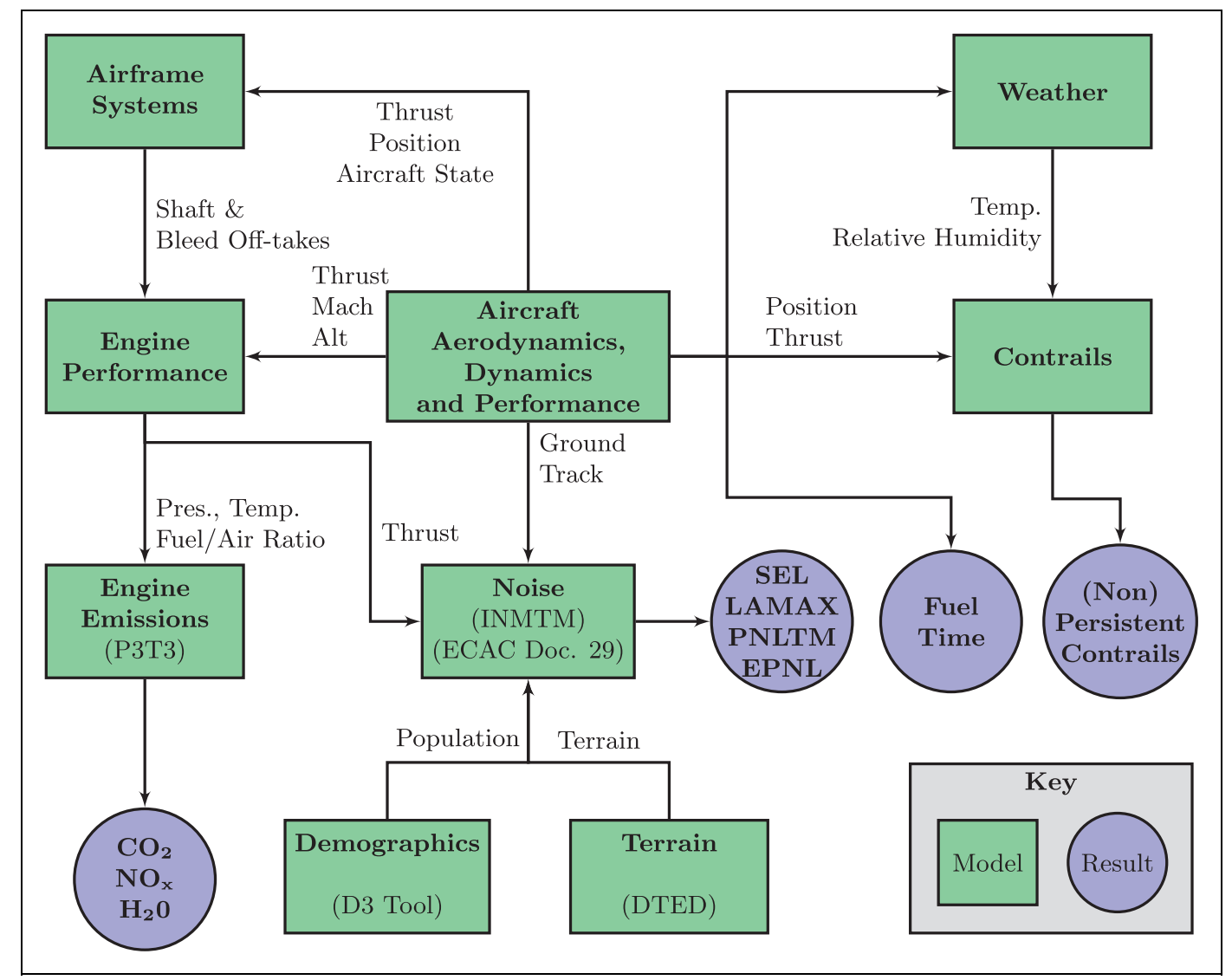

Figure 2. Block diagram of the wider aircraft trajectory optimisation package which this work is a part of.

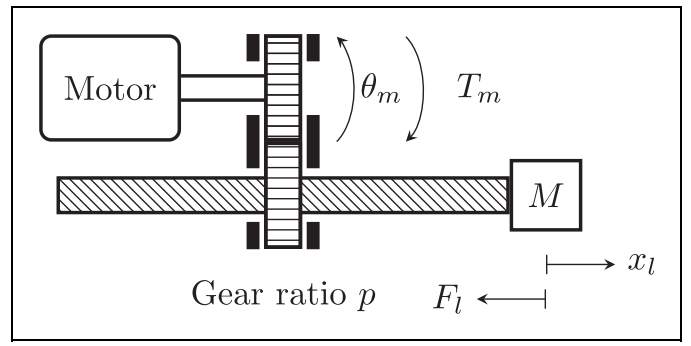

Figure 3. Modelling diagram of an electromechanical actuator.

fast energy consumption estimates to assess different trajectories for global optimality.

Energy losses in the system occur in the electrical (copper and power electronic losses), magnetic (iron and eddy current losses) and mechanical (friction) domains. Friction in a system is important for power consumption modelling because it is the dominant source of mechanical losses in the motor and gearing. Ideally friction coefficients are obtained experimentally, but since this is not available an analytical technique is used to estimate viscous friction. ${ }^{3}$ Electrical and magnetic losses are combined into one equivalent resistance and selected such that the model has a peak efficiency of around 30\%. Such approximations can easily be improved by substituting additional hardware specific data if it is available.

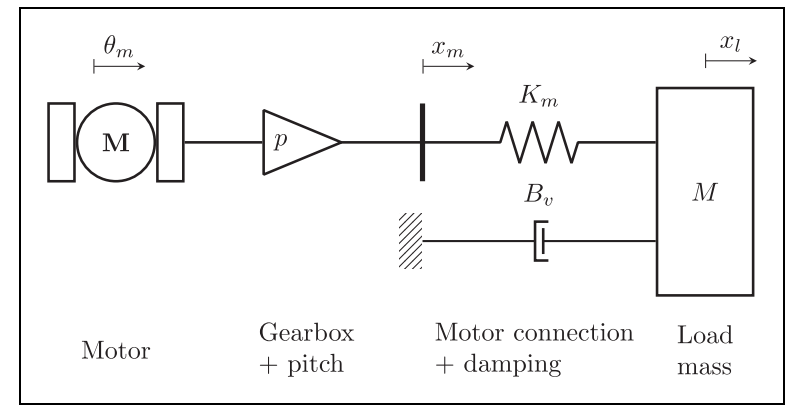

Figure 4. Schematic diagram of EMA used for power consumption modelling.

Validation for the model is achieved qualitatively by writing scripts to assess the open loop performance of the actuator across a range of load forces; the result of which is presented in Figure 5. By recognising that an EMA can be considered for the most part as a motor and gearbox combination, the electrical and mechanical performance variation with load is expected to be dependent on the motor itself (providing the ball screw is of high quality).

The performance data shown in Figure 5 is based on a Portescap 17S78-208 motor ${ }^{4}$ and is recognisable as having speed, current, power and efficiency performance which is typical of a DC motor. The motor is a commercially available unit which provides 


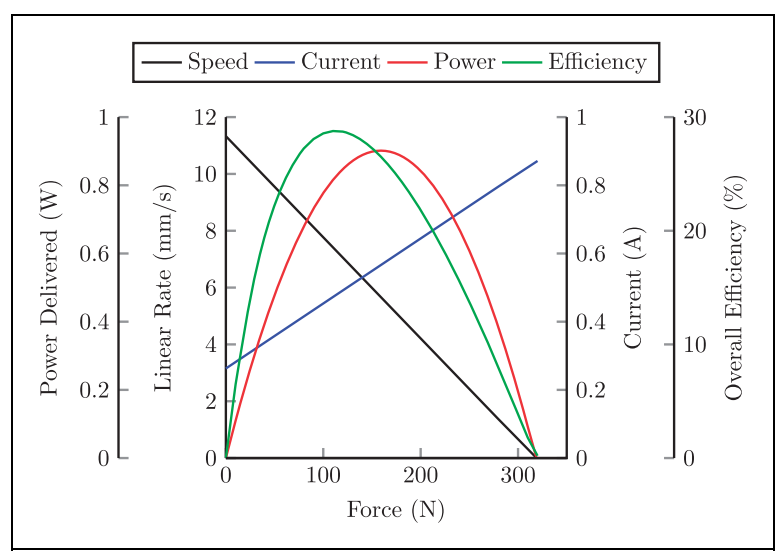

Figure 5. Open loop linear rate, current, power and efficiency against load force at $6 \mathrm{VDC}$ supply voltage.

a good datasheet for parameterising a model, at the cost of being constrained by the existing design and power rating. It is used for all control surfaces and so it is expected to see some 'overdesigned' characteristics in operation.

The linear rate decreases linearly from a maximum at zero load to a minimum of zero at the stall force. There is a linear relationship between current and force which is defined by the torque constant and gear ratio. The power delivered is a product of speed and force, which shows a parabolic characteristic with a maximum at $50 \%$ load and $x$ crossings at zero and stall loads. DC motors are most efficient at high speed (i.e. low torque), because the dominating $i^{2} \mathrm{R}$ heating losses are low. Even at the highest speed, the friction losses are much smaller than the resistive losses are at stall. This can be seen in the figure, where the efficiency increases with decreasing force (and increasing speed) up to a maximum at approximately one-third of the maximum load. When the load force decreases below this the output power and hence efficiency tend towards zero.

Servo-controller. It is important to assess the actuators (motor and mechanical parts) and servo-controller (power electronics and control) separately. The motor and mechanical parts are designed to meet aerodynamic performance requirements, while the servodrive is required to meet the motor requirements. The aim of this work is to focus on meeting aircraft control requirements, so most results are presented without the inclusion of the servo-drive losses to avoid masking the small dynamic powers.

Small servo-drives have a continuous current draw even when idle, which should be considered for aircraft architecture sizing and aircraft level trajectory analysis. To reflect the inclusion of (simplified) servo-control losses, a constant $0.1 \mathrm{~W}$ drain per servocontroller is assumed, applied to five actuators. The results for this assessment will be shown only as a relative overall energy consumption comparison towards the end.

\section{Aerodynamic load estimation and Control Surface Model}

Control surface aerodynamic load estimation can be a complex problem which is often best derived from computational fluid dynamic or wind tunnel investigations. This becomes particularly true at low Reynolds numbers where behaviour is difficult to predict. In order to obtain preliminary aeroload estimates, the same code is applied which has previously been used in the authors' work on transport aircraft. ${ }^{1}$ This software is a MATLAB programmed version of the method published by Roskam ${ }^{5}$ and used by Scholz 6 for preliminary actuator sizing. The limitation of this work is that it does not provide data for Reynolds numbers below 1E6. At the flight condition studied in this paper, the Reynolds number for the tail is around 2E5 and 3E5 for the ailerons. Instead of applying a new method to derive hinge moment coefficients, it has decided to limit the Reynolds number to a minimum of 1E6 and to check the hinge moment estimates fell within the load capacity of the actuators. For this reason the numerical values of the force, power and energy results are not considered to be accurate but the trends formed are representative of the real aircraft.

With the above approximation in mind, the hinge moment coefficients with respect to angle of attack and surface deflection are computed from the lookup tables provided by Roskam and used to calculate an overall hinge moment coefficient using equation (1)

$$
C_{h}=C_{h \alpha} \alpha+C_{h \delta} \delta
$$

The hingemoment torque $T_{H M}$ is then calculated using equation (2), before being converted to a force by using the control surface moment arm. It should be noted that these two formulae must be computed at every time step to generate transient aeroloads on the control surfaces, but the derivatives $C_{h \alpha}$ and $C_{h \delta}$ remain constant

$$
T_{H M}=C_{h} q A_{w} \bar{c}
$$

Once the load forces on the actuators have been estimated, the linear actuators' outputs are converted to rotational motion of the control surfaces through a moment arm. The actuator installation is shown in Figure 6.

The control surface angle $\delta$ is calculated using the cosine rule in equation (3)

$$
\delta=\cos ^{-1}\left(\frac{b^{2}-c^{2}-a^{2}}{-2 a c}\right)-\beta_{0}
$$

\section{Aircraft model (6-DoF)}

The aircraft model used in this work represents the Aerosonde weather reconnaissance and remote 


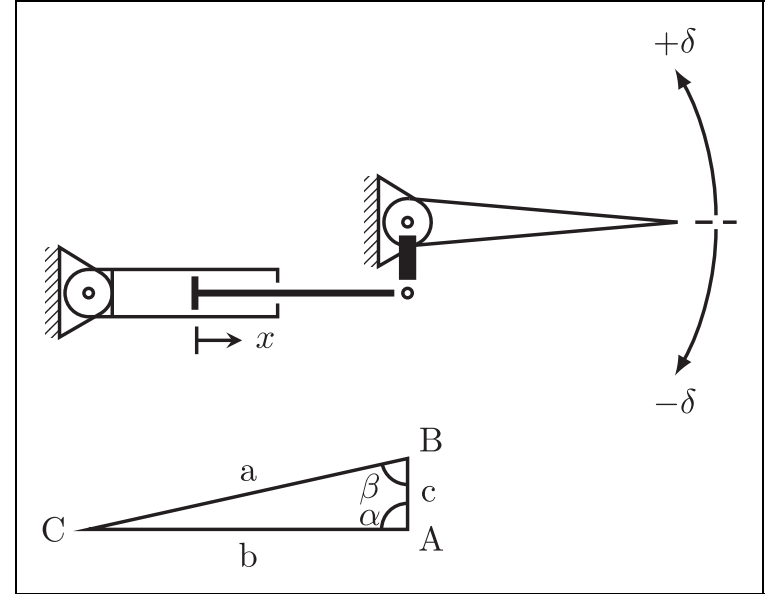

Figure 6. Actuator installation schematic.

sensing UAV. ${ }^{7,8}$ Aerosonde is a twin-boom, inverted v-tail UAV of wingspan $3.6 \mathrm{~m}$ and gross mass $17 \mathrm{~kg}$. It has a payload capacity of $3.5 \mathrm{~kg}$ and $75 \mathrm{~W}$. It is modelled as a variable mass, rigid body object which experiences aerodynamic, propulsive and gravitational forces. The motion of the aircraft is defined by the position and velocity of its centre of gravity, along with the orientation angles and body rates of a body fixed axis system with respect to an inertial reference frame. Newton's second law is used to describe the linear and angular motion of the aircraft using equations (4) and (5), which are based on the derivation provided by Beard and McLain ${ }^{8}$

$$
\begin{aligned}
& \frac{d \boldsymbol{V}_{b}}{d t}=\frac{1}{m} \boldsymbol{F}-\boldsymbol{\omega}_{b} \times \boldsymbol{V}_{b} \\
& \frac{d \boldsymbol{\omega}_{b}}{d t}=\frac{1}{\boldsymbol{J}}\left[\boldsymbol{M}-\boldsymbol{\omega}_{b} \times \boldsymbol{J} \boldsymbol{\omega}_{b}\right]
\end{aligned}
$$

The assumptions made in constructing the 6-DOF model are the following

- The atmosphere model is the International Standard Atmosphere, considered at rest relative to the Earth.

- Temperature, pressure and density are considered to be a function of altitude.

- The aircraft is considered as rigid and with a right-left plane of symmetry.

- Forces acting on the centre of gravity are the aerodynamic forces, thrust and weight.

- Gravitational acceleration is considered as a constant $9.81 \mathrm{~m} / \mathrm{s}^{2}$.

The force and moment vectors are computed using aerodynamic coefficients and an engine model for the Aerosonde provided in the textbook by Beard and McLain. ${ }^{8}$ Equations (4) and (5) are implemented in Simulink using Aerospace Blockset components for computational efficiency, where the outputs are integrated twice to obtain linear and angular velocities

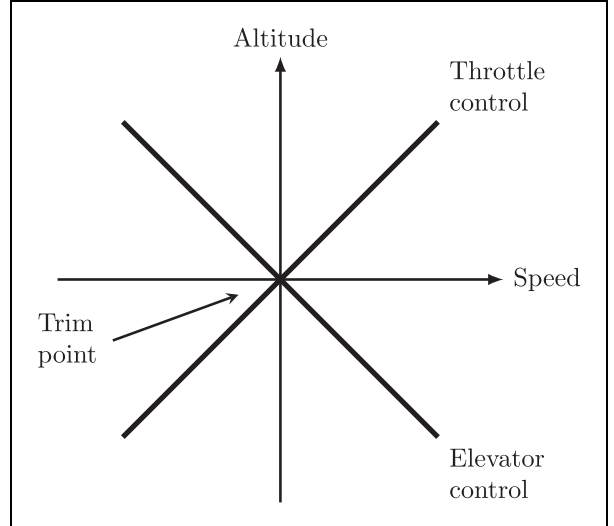

Figure 7. Longitudinal energy of a trimmed aircraft in flight. ${ }^{10}$

and positions. The result is an open loop Aerosonde model which executes many times faster than real time and can provide control surface position commands to the actuator models.

\section{Automatic flight control system}

One fundamental problem with conducting aircraft performance analyses using a regular 6-DOF (noneinverse type) is that the model requires a flight control system (FCS) to ensure it follows the chosen trajectory. A forward 6-DOF model uses aerodynamic coefficients, driven by the control inputs to generate forces and moments which are used by the equations of motion to derive time varying attitudes and positions of the aircraft. In order to control the position of the aircraft, the loops must be closed to obtain good control of the inputs in order to manoeuvre the aircraft as required.

This is classically achieved using a selection of single input-single output (SISO) controllers which control a single aircraft DOF with a single control effector. This technique is widely used, but as demonstrated by Lambregts; ${ }^{9}$ this control scheme produces unnecessary movement of the control inputs because a real aircraft has strongly coupled axes of motion which are not naturally taken into account. Achieving a level acceleration or constant speed climb from steady level trimmed flight requires synchronised changes in both the throttle and elevator positions, due to the cross coupling between the two controls (shown in Figure 7).

In a classical autopilot the elevator and throttle controls are implemented with two separate loops which have no knowledge of each other's states. Lambregts developed an integrated multiple inputmultiple output (MIMO) total energy control system (TECS) which sought to unite throttle and elevator controls into a single aircraft energy state controller. ${ }^{9}$ Since the referenced document provides an excellent explanation and derivation of the equations, they will not be replicated here. In summary, the aircraft's total energy (gravitational potential energy + kinetic 
energy) is controlled using the throttle, while the elevator is used to transfer energy between the two forms. The technique has been successfully trialled on several aircraft from UAVs (NASA Condor ${ }^{10}$ ), to manned general aviation aircraft ${ }^{11}$ and large transport aircraft (Boeing 737). ${ }^{12}$

In an analogous fashion, the lateral motion can be controlled using energy principles in a total heading control system (THCS) as shown initially by Bruzzini on an F-15. ${ }^{13}$ This synchronises the ailerons and rudder to allow the aircraft to execute coordinated manoeuvres using a MIMO structure, facilitating banked turns at constant sideslip or sideslip changes at constant heading. The former is useful in normal manoeuvres for keeping the sideslip angle at zero while changing heading between flight path segments. The latter is useful during crosswind landings or in the event of asymmetric flight due to engine failure. It should be noted particularly that all these functions are provided by a single MIMO control structure with THCS; a classic SISO autopilot would require several layers of controllers with logic switching to accomplish these tasks satisfactorily.

TECS/THCS has two main benefits to this work which will be discussed in the following paragraphs:

- Increased simulation speed achievable when using a 6-DOF model.

- Point mass representation of the aircraft dynamics at the outer loop inputs/outputs.

When a 6-DOF model is controlled using TECS/ THCS, larger timesteps can be used for the numerical solver used to simulate the model. This is directly related to the reduction in root mean square ( $\mathrm{rms}$ ) surface deflections noted in the prior work by Bruce. ${ }^{14}$ The author found, for example, a reduction in elevator activity in turbulence from $0.81^{\circ} \mathrm{rms}$ to $0.16^{\circ} \mathrm{rms}$ for a simulation of a Boeing 737 aircraft (without penalising velocity or altitude tracking). Lower activity translates to less hunting as the various SISO control loops fight each other and significantly reduces oscillations. From a speed of execution perspective, TECS/THCS allows for reliable, high-speed simulation of a controlled 6-DOF model across its flight envelope.

The second benefit of TECS/THCS to this work is the masking of high frequency aircraft dynamics behind low bandwidth outer control loops. As noted by Faleiro and Lambregts, ${ }^{15}$ this behaviour lends itself admirably to integration with flight management computers which consider trajectory management as a point mass kinematic problem. The outer loops of TECS/THCS are derived from point mass kinematic equations, making TECS/THCS an ideal candidate for trajectory following whilst ensuring the aircraft conforms to airspace safety requirements. Simply stated, a TECS/THCS control system with suitably designed aircraft specific inner loops will follow any trajectory commanded of it with less tracking error and control effort than simple SISO controllers (providing the desired trajectory has achievable climb and turn rates). Since actuator power consumption can depend heavily on the controllers used, TECS/ THCS is selected as the ideal FCS to follow trajectories with the minimum control energy.

\section{Guidance system}

All experiments are conducted with the FCS loops closed, such that the aircraft will maintain any commanded speed, altitude, heading and sideslip angles (within the aircraft's flight envelope). In order to investigate manoeuvres systematically, it is important to provide some guidance algorithm to enact manoeuvres consistently. This can be as simple as commanding a step change in heading at a fixed instant in time during the simulation; the problem with this is shown in Figure 8 where the aircraft can clearly be seen to exit the heading change manoeuvre along different parallel ground tracks as a result of different turn rates. The figure is generated by disconnecting the guidance controller and triggering a heading change after $1 \mathrm{~km}$ of flight. This effect is quite obvious but it makes it difficult to compare numerical results since aircraft following each route will exit the manoeuvre on a distinctly different ground track; not something which is very useful when considering a transport aircraft that needs to depart from point $\mathrm{A}$ and arrive at point $\mathrm{B}$ or a UAV which has to traverse a specific area on the ground.

This behaviour is intuitive; the solution is to define a 'fly-by' waypoint some distance in front of the aircraft starting point which can be used to define a specific entry and exit flight path. The turn is then initiated at a distance $d$ from the waypoint, which is computed from the aircraft's forward speed and the turn rate; leading to a 'filleted' turn between two flight path segments (or airways). To achieve turns with no overshoot requires knowledge of the aircraft 'time to bank' (TTB) which is essentially a measure of how long (in time or distance) before reaching the turn point $d$ that the aircraft must start banking so that it crosses $d$ at the desired bank angle/turn rate. In this work TTB compensation is left out for simplicity and to avoid distorting the results by adding layers of complexity induced by the guidance algorithm.

The guidance algorithm for this task is formulated from the straight segment and orbit following textbook method provided by Beard and McLain. ${ }^{8}$ This algorithm defines the manoeuvres using the turn radius, by computing the indentifying coordinates of a tangential circular orbit connecting two intersecting straight waypoint paths. The straight paths are defined beforehand to specify the route to be followed. Transitions between the different manoeuvre points are detected using half plane triggering to ensure robustness, avoiding the pitfalls of radial 


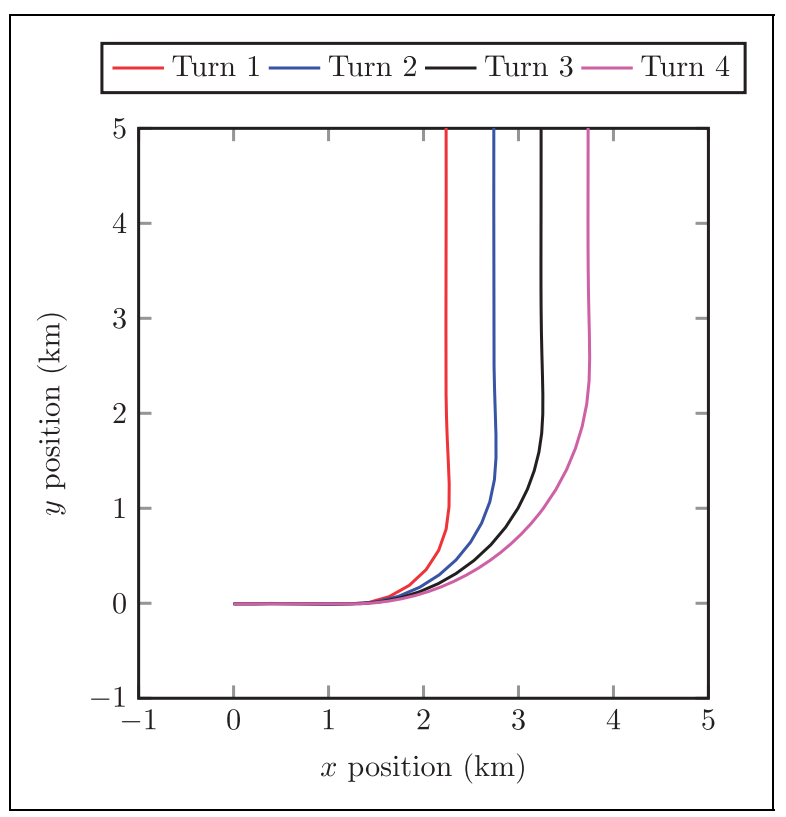

Figure 8. Trajectories flown by aircraft turning $90^{\circ}$ at a range of heading rates (with no guidance system).

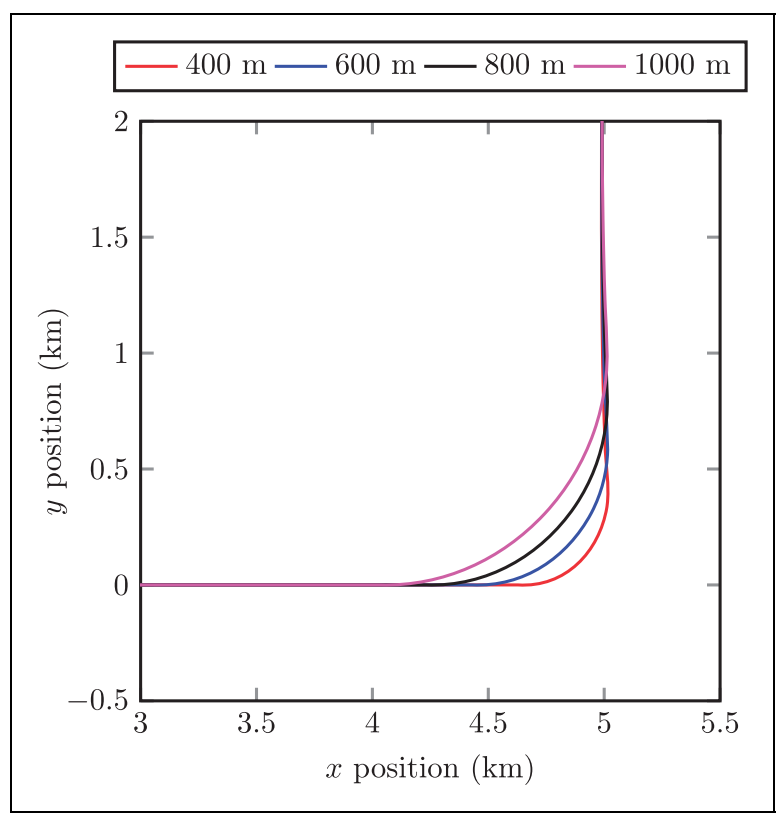

Figure 9. Trajectories flown by aircraft turning $90^{\circ}$ at a range of heading rates (with guidance system).

waypoint detection methods in small UAVs. The complete guidance algorithm is programmed as a statemachine using Simulink's Stateflow software, which compiles the code to allow fast execution and natural integration with the Simulink based aircraft model.

A demonstration of the guidance algorithm is shown in Figure 9 for a $90^{\circ}$ turn. The aircraft evidently anticipates the prescribed turn rates and initiates the turn at such a distance from the first waypoint (located at $(5,0)$ ) in order to intercept the next flight path segment with minimum error. This is a clear advantage of the method since it allows forward simulation models (such as those used in this paper) with controllers to be assessed while tracking any achievable trajectory.

\section{Simulation method}

The simulation experiment performed in this paper is designed to mimic the application of the simulation framework to a simple trajectory optimisation problem. An aircraft needs to turn $90^{\circ}$ to the left and has several options of which path to follow to get there. For this paper, the trajectories to be assessed are selected to be a banked turn at a series of different turn rates.

The method is structured in the following way. A single aircraft model is created and equipped with a TECS/THCS FCS and waypoint guidance system. This model is self contained, can be executed on its own and will follow a single list of waypoints.

The guidance algorithm is supplied sequentially with each possible route and the model is executed using the ode23tb solver. The aircraft and actuator state data from each trajectory is stored for later processing. Once every route has been performed the data is combined and plot to perform a visual analysis.

To expand on the first experiment and demonstrate the operation of the model in more realistic situations, a longer flight with the addition of a Dryden gust model is conducted.

The key requirements for high-speed simulation are:

- Use of solvers for stiff systems; the bandwidth of the actuator motor controls are significantly higher than that of the slow aeroplane dynamics.

- Long maximum timesteps for the variable step solver (ode23tb with a 1-s max. timestep is used).

- Careful adjustment of solver absolute and relative tolerance to allow automatic adjustment to long timesteps in periods of low activity (straight segments) and suitably small timesteps during periods of high activity (during manoeuvres).

- Vectorise multiple actuators into a single model.

- Minimise control oscillations from well-tuned controllers.

- Use of MATLAB's Parallel Computing parfor loop in the main program control loop so one complete model/trajectory can be computed on each processor core/computer.

- Initialise model from a trimmed operating point for all states.

To expand on the last point; the aircraft model is trimmed without FCS or actuator models for the development stage while the flight controller, actuators and aeroload estimator are integrated. This trims the aircraft inputs and states so it is flying straight and level at a chosen altitude and airspeed. After integration is complete, continuing to initialise the complete model this way causes a 
severe slowdown at the start of the simulation. This is a result of the actuator models initialising with zero load on them; motors stationary and with no torque being generated. At the same instant in time $(t=0)$, the aeroload estimator produces a non-zero load force on the ailerons, elevator and rudder (if sideslip angle $\beta \neq 0$ ) which requires a step change in motor torque requirement. The resulting damped oscillations that occur as the actuator controllers track towards their new (loaded) steady state operating points requires small timesteps to capture the motion.

To avoid this, Simulink's operating point search function is used to capture the values of all states after a long simulation time (1000s is used) which are then used to initialise the complete model from a trimmed operating point. For this part of the process the guidance system is disconnected and the aircraft model is initialised from the previous state of trim. By giving the simulation a long time to run, the FCS maintains the aircraft flying at the previously specified flight condition whilst the actuators adjust to the steady state loaded conditions. Note that for variable mass aircraft models the fuel mass should be fixed to ensure that the state values captured at the end of the simulation are valid for the aircraft mass at the start of the simulation. Some values must be manually set to zero, such as the aircraft position and actuator energy integrators, for correct operation during the main experiment.

\section{Results}

The first plot in Figure 10 describes the variation in attitudes required to execute the various turn rate manoeuvres. The bank angle increases in proportion to the increasing turn rate up to a maximum of $12^{\circ}$ and the nose is raised in the second plot to maintain a constant altitude. The bottom axes show the aircraft turning at various rates toward the new heading. Cross coupling is evident between all three axes of motion, particularly strongly during the fastest turn of $400 \mathrm{~m}$ radius.

The airspeed and altitude plots of Figure 11 show the departure from trim values during the manoeuvres. TECS achieves a worst case airspeed error of less than $\pm 0.04 \mathrm{~m} / \mathrm{s}$ and a worst case altitude tracking error within $+0.04,-0.08 \mathrm{~m}$.

The control inputs to the 6-DOF model generated by TECS are shown in Figure 12. As expected, a higher turn rate causes a higher deflection from trim for all control surfaces. Transient synchronisation is evident between the ailerons and rudder and also the elevator and throttle. A large spike in the responses is evident at $220 \mathrm{~s}$ (for the $400 \mathrm{~m}$ turn) when the guidance algorithm switches back to straight line tracking after the orbit. Boxplots have been included to show the statistical properties of the surface velocities throughout the manoeuvre. This includes maximum, minimum and median, as well as the range between

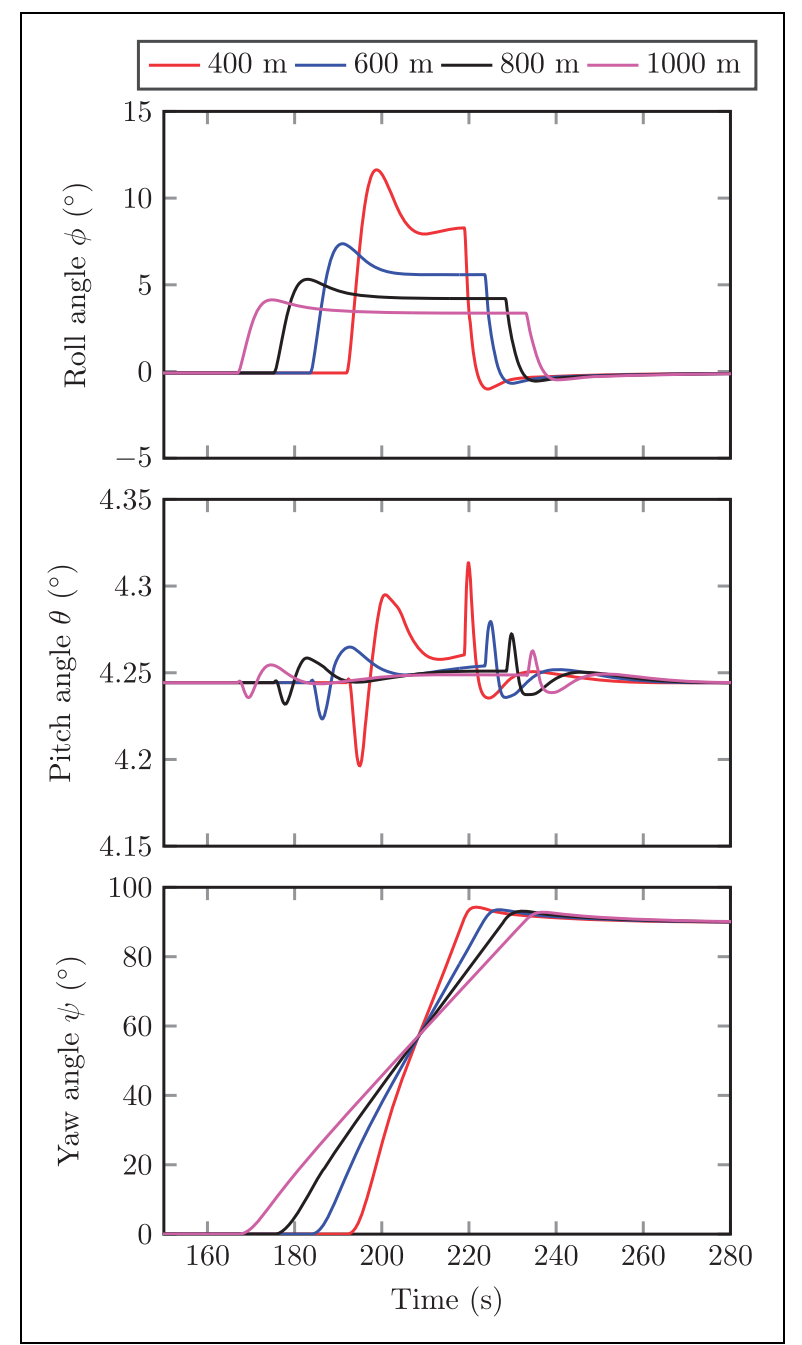

Figure 10. Euler angles (top: roll $(\phi)$, middle: pitch $(\theta)$, bottom: yaw $(\psi))$.

the 1st and 99th percentiles of velocities over the course of the trajectory.

The aerodynamic loads exerted on the control surfaces are shown in Figure 13; in this figure the two ailerons are shown separately to highlight the asymmetric load on each surface. The actuator load plots are proportional to the deflection plots in the previous figure and show the same trends.

The instantaneous electrical powers demanded by the actuators are shown in Figure 14. The ailerons demand a maximum of $30 \mathrm{~mW}$ to enter the turn and a peak up to $3.8 \mathrm{~W}$ to exit it and return to straight line tracking. The elevators require a maximum of $0.17 \mathrm{~mW}$ to maintain attitude on entering the turn and a peak power of $0.37 \mathrm{~mW}$ to exit the turn. The rudder has a peak load of $0.37 \mathrm{~W}$ and a negligible power during the constant rate turn.

A metric of average power is used to compare the different manoeuvres in Figure 15, by integrating the positive instantaneous power to compute energy demand and dividing by the time for each trajectory. Every surface shows a parabolic increase in energy with decreasing turn radius. The straight line mean 


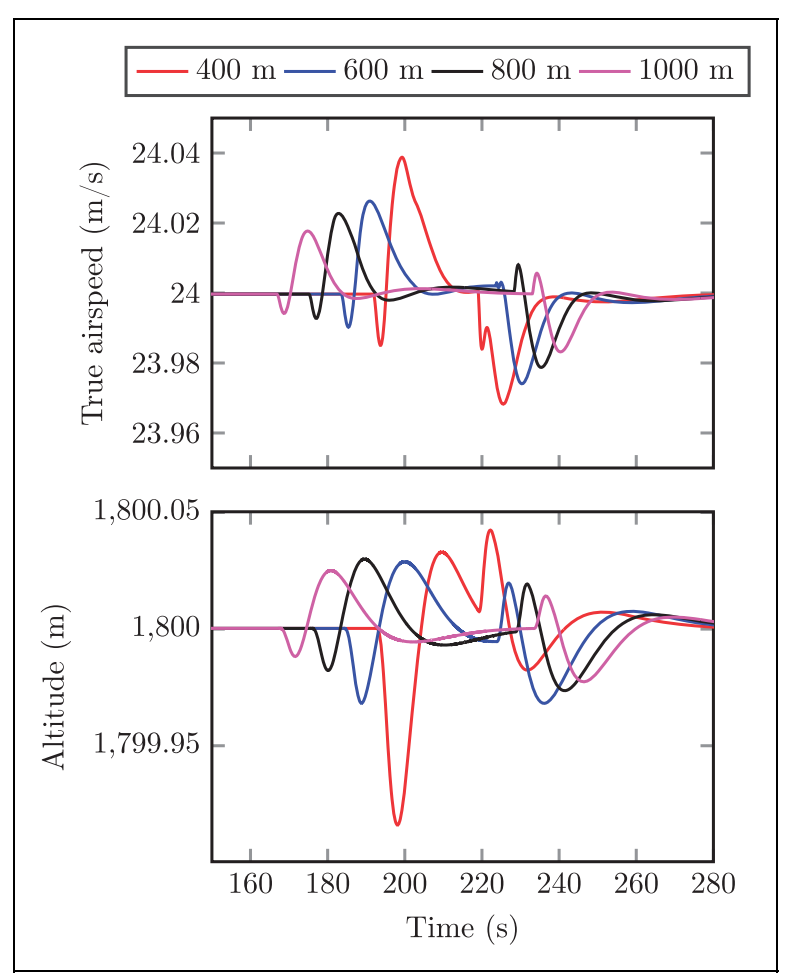

Figure II. Top: true airspeed, bottom: altitude.

power for the ailerons, elevator and rudder is $4.47 \mu \mathrm{W}, 2.53 \mu \mathrm{W}$ and $0.04 \mu \mathrm{W}$, respectively. The maximum mean power for each is $393.90 \mu \mathrm{W}$, $3.33 \mu \mathrm{W}$ and $48.31 \mu \mathrm{W}$, increasing by factors of 88 , 1.3 and 1208 from their straight line averages.

The aileron and rudder show an unusual trend, where smaller heading changes require a higher average power than a larger turn (at each turn radius). The elevator generally follows the opposite trend with the exception of the $30^{\circ}$ case. To investigate the reason for this, the time-series energy plots for the $400 \mathrm{~m}$ manoeuvres are extracted and plot in Figure 16. The aileron and rudder axes show the energy required to enter the manoeuvre is lower than that needed to leave it, while the elevator requires more energy to enter the turn. The elevator plot shows a linear increase in energy consumed over time for all cases.

The total energy consumption for all five actuators is presented in Figure 17, which shows that the average power demand for the $400 \mathrm{~m} 90^{\circ}$ turn increases by 12.8 times over the $1000 \mathrm{~m}$ turn radius, when only the motor is considered. When the constant steady state power loss of the servo-controllers is included, the same comparison results in a factor of only 1.001.

The components of mechanical power for the manoeuvres are shown in Figure 18, where higher turn rates are seen to require higher actuator forces and velocities (up to $506 \mathrm{mN}$ and $7.58 \mathrm{~mm} / \mathrm{s}$ maximum). The plots all tend towards a location on the zero velocity line and spend the majority of time exposed to a positive force.

Following on from the $90^{\circ}$ turn results are the outcomes of the longer distance mission with turbulent

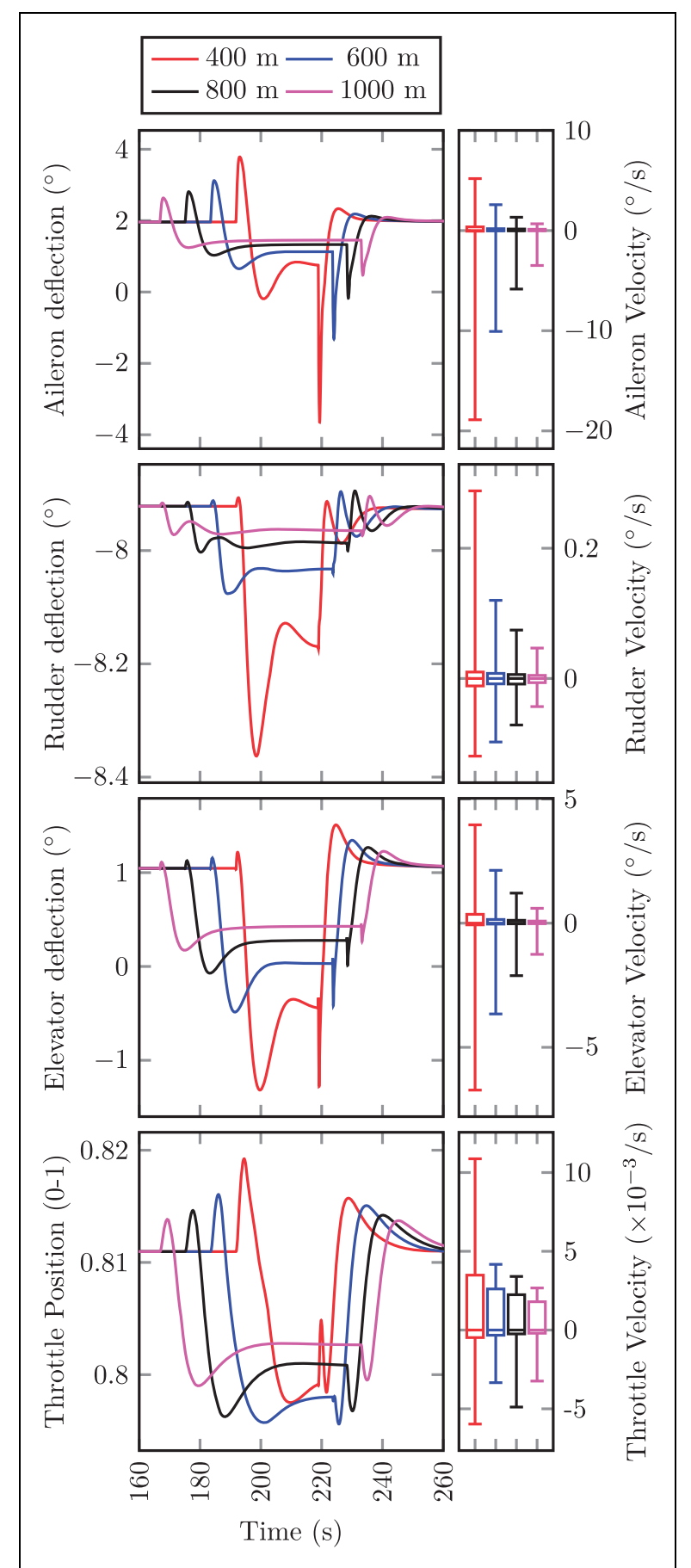

Figure 12. Control surface deflections (left) and a statistical overview of the control velocity throughout the trajectory (right), showing the maximum, minimum, median and Ist and 99th percentiles. (Top: aileron, upper middle: rudder, lower middle: elevator, bottom: throttle).

conditions. Figure 19 shows the planned route and the actual trajectory achieved by the flight control and guidance systems. There is very little deviation, with no noticeable steady state tracking errors or manoeuvre overshoot.

Figure 20 shows the output of the simulation tool in windy conditions, allowing a cost factor for the current trajectory to be computed. The figure shows a zero 


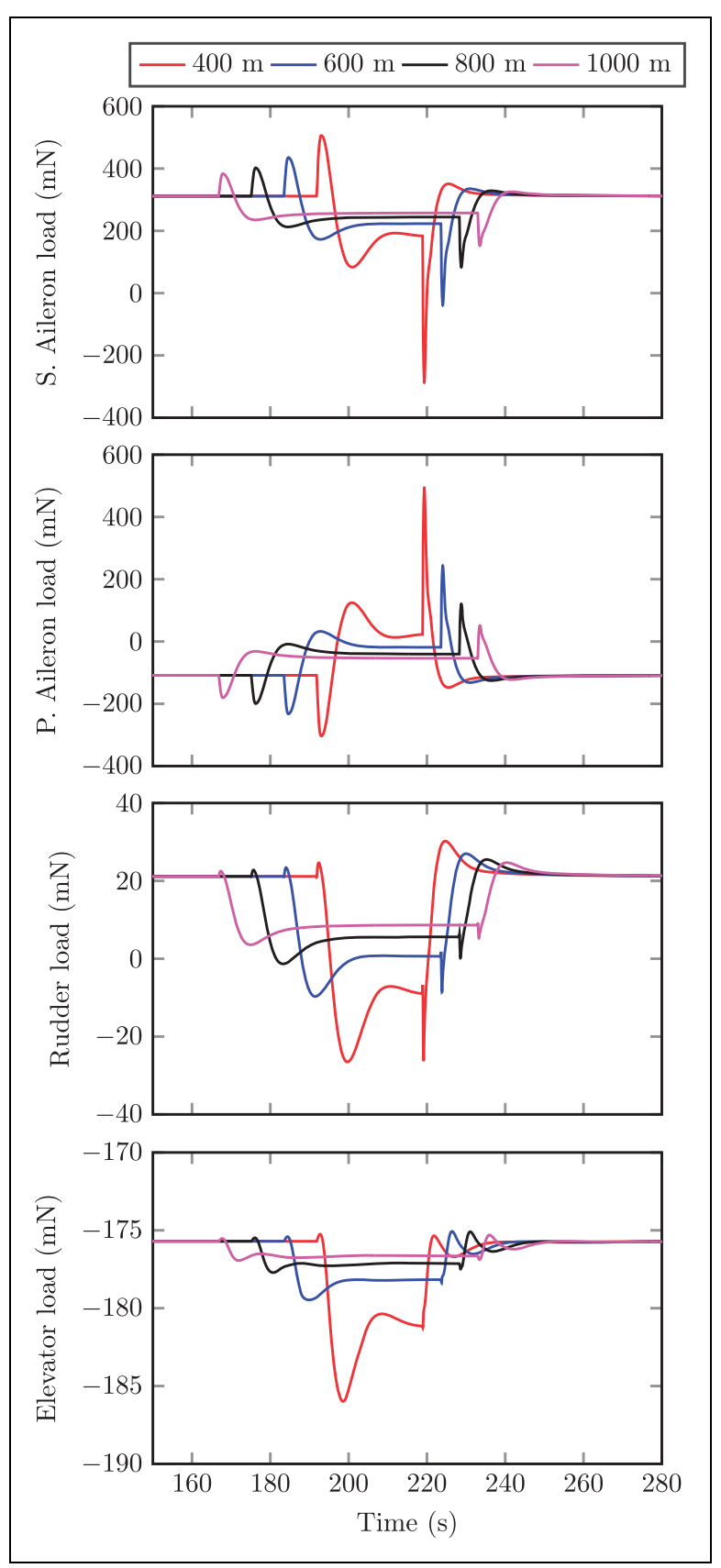

Figure 13. Aerodynamic forces on surfaces (top: starboard aileron, upper middle: port aileron, lower middle: rudder, bottom: elevator).

mean velocity and non-zero load force, with a maximum force and velocity of $1.57 \mathrm{~N}$ and $23.89 \mathrm{~mm} / \mathrm{s}$.

\section{Discussion}

The expected trend is to see larger control surface deflections and higher powers as the turn rate (and bank angle) increases. When conducting the experiment with a full 6-DOF model, this relationship can be quantitatively investigated as will be discussed in this section. It should be noted that the motor model used for the analysis is parameterised from a unit which is oversized for the application, but the

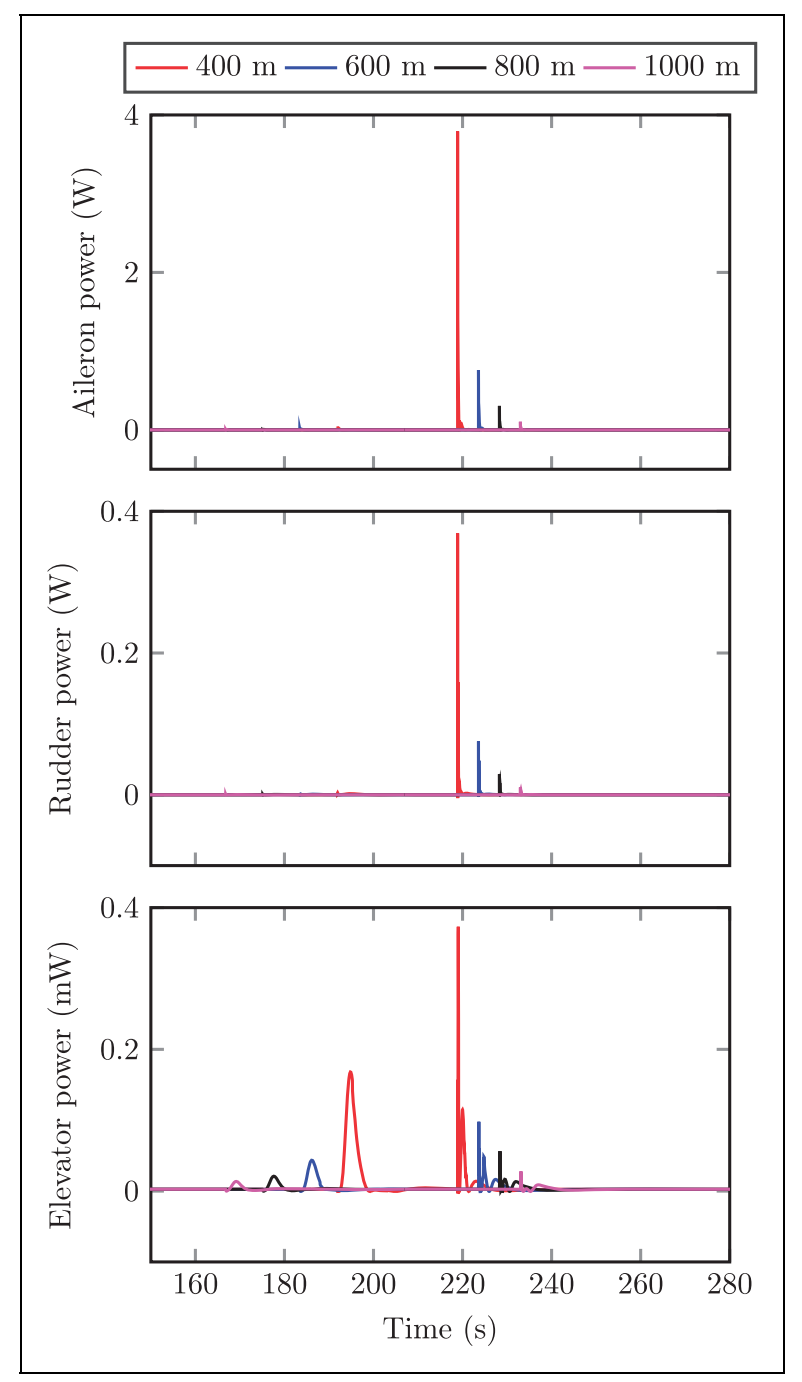

Figure 14. Instantaneous electrical power (top: aileron, middle: rudder, bottom: elevator).

intention is to display the performance of a complete system in a given configuration to drive further refinement with additional quantitative knowledge. The aim of this paper is not to accurately describe a specific existing aircraft.

An overshoot is exhibited on the heading angle response in Figure 10 which is attributed to the guidance system not taking into account the TTB; as the turn gets faster the bank angle increases along with the TTB (and level out). This causes increasing overshoot as the turn rate increases on the uncompensated guidance algorithm which could be reduced with additional functions to anticipate the TTB.

Synchronisation is seen between the aileron and rudder controls in Figure 12 which demonstrates the correct transfer of energy between the roll and yaw axes. The initial impulse required to induce the manoeuvres are seen to increase exponentially as the turn rate increases. While perfectly understandable, these large deflections are in the opposite direction to that needed to cause the roll desired. In fact, they are a result of the sideslip angle cancellation function which THCS provides. 


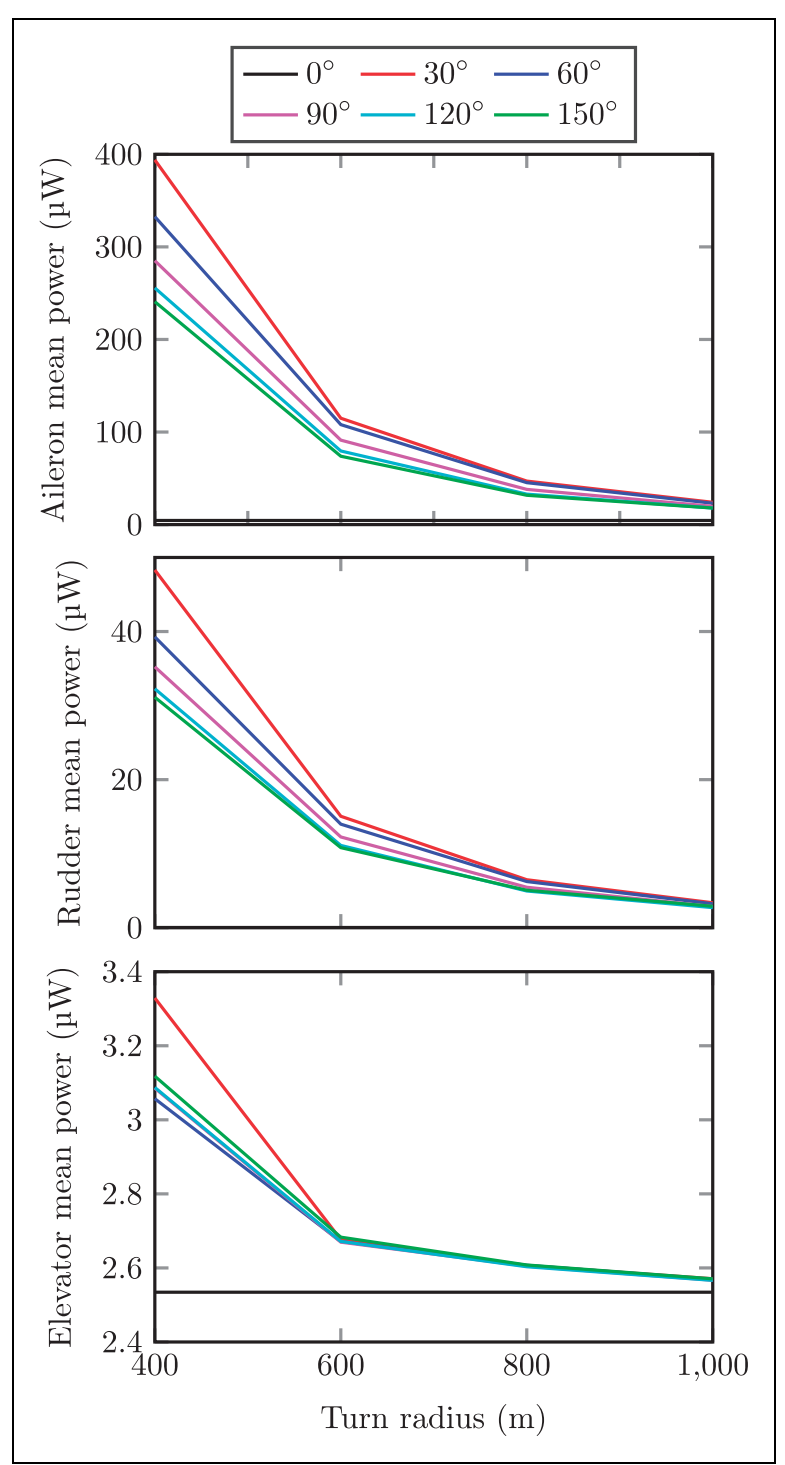

Figure I5. Total motor electrical energy used (averaged by distance travelled) (top: aileron, middle: rudder, bottom: elevator).

On the Aerosonde, because of the torque induced by the single propeller, the trim condition requires the deflected ailerons and rudder seen in Figure 12 to maintain steady level flight. On an aircraft with two contra-rotating propellers or jet engines, the aileron deflection angle would be zero and as such the load force on each surface would be numerically equal once the aircraft had returned to steady level forward flight.

The boxplots in Figure 12 highlight that the maximum and minimum velocities are outliers with the actuators spending the majority of their time at low velocities, however the supply components must still be designed to meet these demands. As will become more clear in the next figures, these peaks cause a direct increase in motor peak power consumption.

The aerodynamic load forces shown in Figure 13 are proportional to the control surface deflections in Figure 12; this is expected since the aerodynamic load

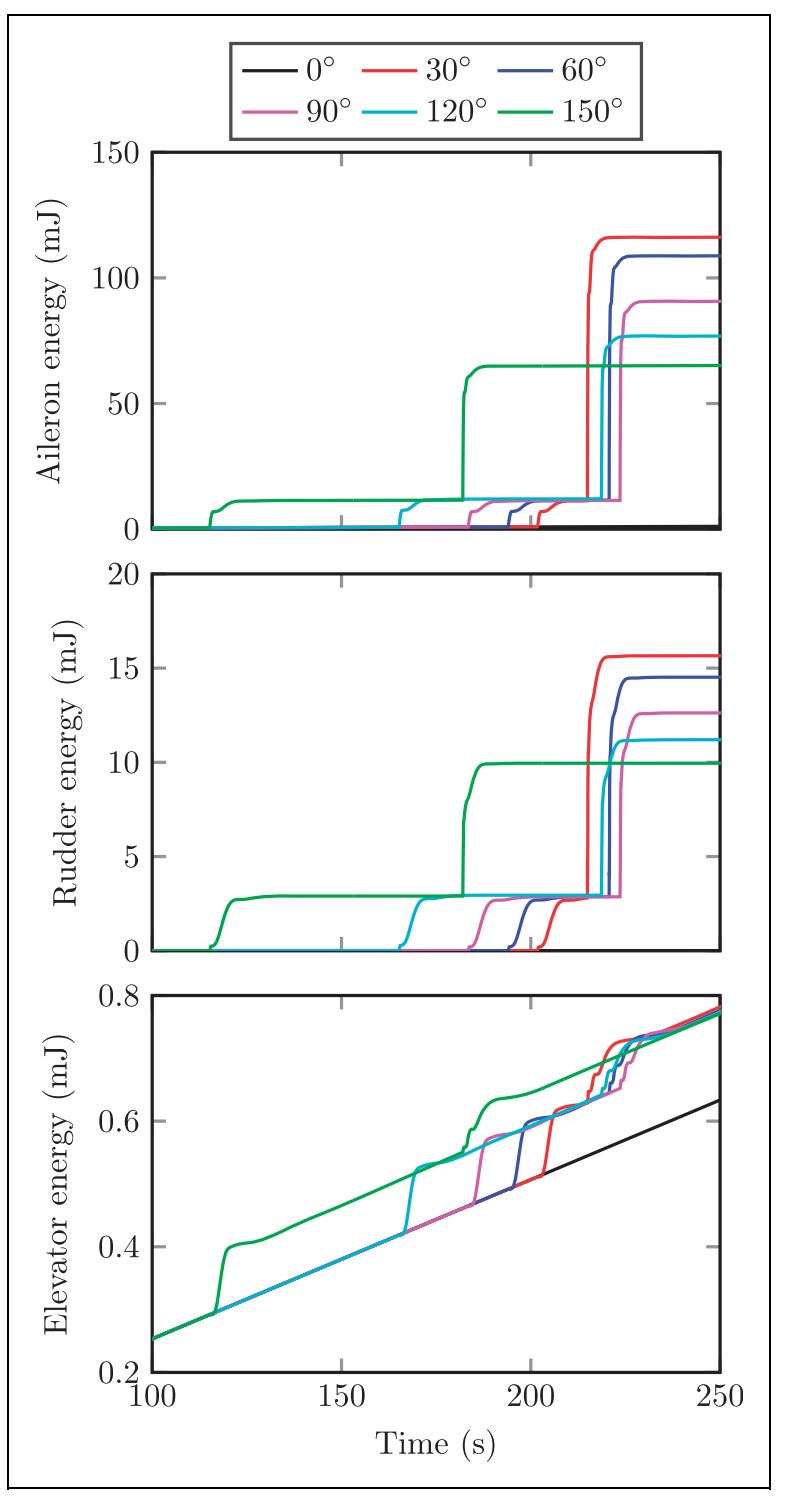

Figure 16. Energy consumption of the actuators over time, during a $400 \mathrm{~m}$ radius turn.

estimation tool produces a linear approximation against surface angle.

Figure 14 demonstrates the benefit of approaching the actuator power estimation task using a 6-DOF model; there is a lot of dynamic behaviour which could not be captured using steady state approximation methods. In terms of quantifying trajectory optimality, these plots lend insightful knowledge into the average and peak power required compared to 'typical duty cycle' methods. As such, use of this method can have positive cumulative implications on the aircraft electrical sizing cases. It shows how low the average power can be; for example energy conservation between the two aileron actuators means one actuator requires more power to bank, but it is counterbalanced by an equal reduction in that supplied to the opposite actuator.

The parabolic increase in energy consumption with reducing turn radius in Figure 15 is attributed to the 


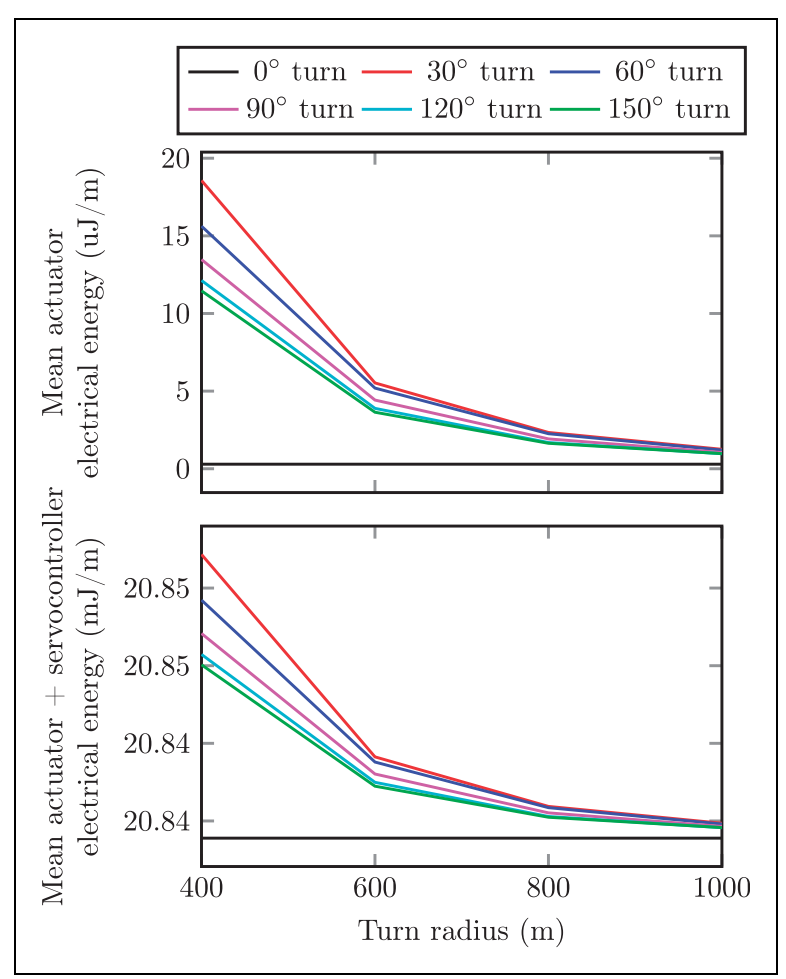

Figure 17. Effect on electrical energy consumption of servocontroller (averaged by distance travelled).

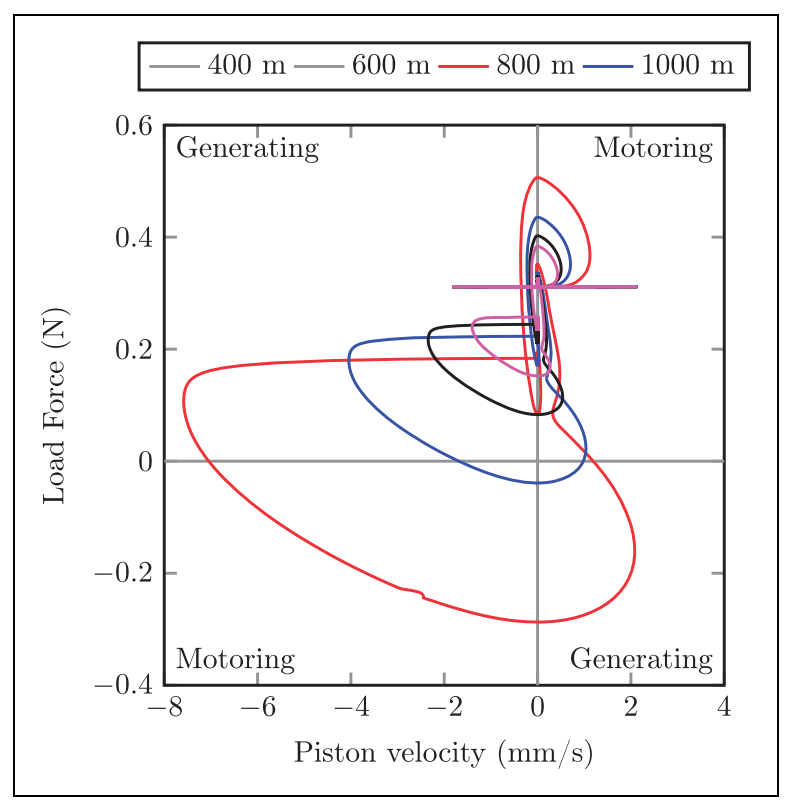

Figure 18. Force-speed plot of the starboard aileron actuator during the $90^{\circ}$ turn.

higher bank angles required, leading to increased excitation of the cross-coupled axes of aircraft motion. Hence, greater actuation power is needed to maintain the aircraft along the desired trajectory.

Similarly, the flight controller must achieve higher aircraft rotational accelerations to achieve smaller heading changes (and to correct trajectory tracking errors) at a given turn radius. This explains the trend of increasing average power demand for decreasing heading changes seen in the aileron and elevator plots. According to the technique used to compute average power in Figure 15, the elevator trend is the opposite (higher average power for larger heading changes) with the exception of the $30^{\circ}$ turn.

The $30^{\circ}$ turn departs from the trend set by the other manoeuvres since the aircraft cannot physically roll fast enough to achieve the required bank angle in such a short turn (see Figure 10), becoming particularly problematic at the higher turn rates. The longitudinal and lateral flight controllers interact to drive the power demand of the elevator up (relative to the trajectory time) at the $30^{\circ}$ turn.

The elevator average power trend is at odds with the pattern of overall energy consumed by the elevator in Figure 16, which shows the $150^{\circ}$ turn using the least, and the $30^{\circ}$ turn using the most. The difference between the values is so low that when divided by the trajectory time to compute average power, the results are distorted as a result of imperfect trajectory tracking.

The average power demand from Figure 15 provides little detail on how the manoeuvre consumed energy and therefore how the flight controller or trajectory could be optimised. Figure 16 plots the energy consumed over time for the three actuators; the aileron and rudder axes show the FCS turns consistently into the manoeuvre, using the same amount of energy to achieve a $400 \mathrm{~m}$ radius banked turn. The roll out overshoot from the shorter turns tend to be larger than those for large heading changes, requiring more power to correct the course towards the next straight line segment.

The inclusion of the servocontroller losses in Figure 17 highlights that from an energy perspective, flight control actuators have a minimal effect on the overall demands of a small UAV power architecture. The additional consumptions to manoeuvre may not be significantly higher than the general consumption of the devices while flying straight.

Figure 18 provides a neat method to characterise the operating quadrant of the actuator, while also displaying information on the magnitude of the force and velocity through a mission. The data in the figure demonstrates the ability of the method presented in this paper to analyse and compact a trajectory into one data plot. These force-speed plots can have spatial statistics applied to quantify a penalty factor for a particular flight path, which then feeds back to the optimisation process.

The previous figure shows the force-velocity data of a single banked turn manoeuvre, which has been the focus of this paper. The method of course allows more in-depth investigations to be performed; for example, longer trajectories and the inclusion of turbulence. A demonstration of this capability is included as the final piece in this paper; Figure 19 shows the flight path of the UAV performing 


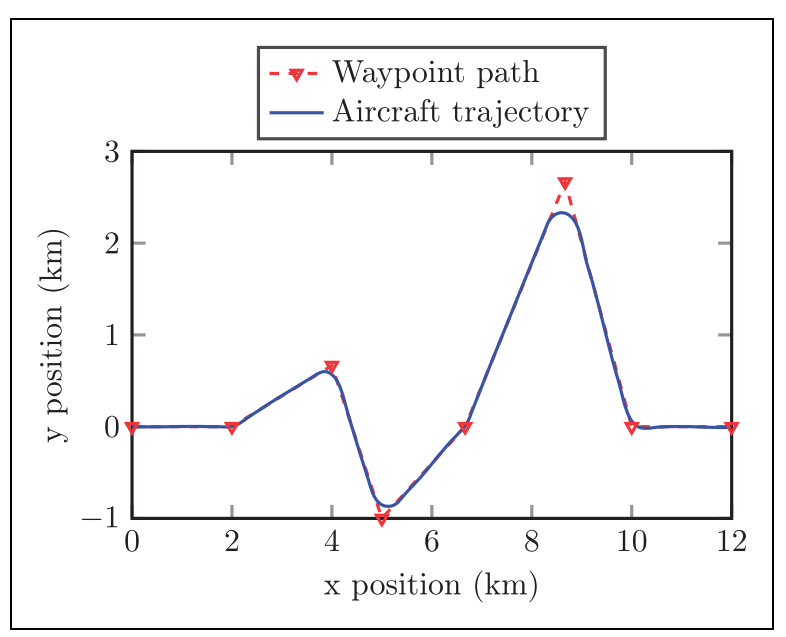

Figure 19. Trajectory followed by the Aerosonde in turbulent conditions.

arbitrary heading changes and finishing a distance $12 \mathrm{~km}$ from the starting location in windy conditions. The planned route is user specified and provided to the guidance algorithm, with the first coordinate and segment bearing also being used to initialise the aircraft model position. The guidance algorithm and flight controller successfully maintain the aircraft flying along the predefined route and execute filleted turns, even when exposed to turbulent wind sources.

The aircraft is subjected to turbulence provided by a Dryden gust model which adds a filtered white noise velocity vector to the aircraft's velocity vector. The aim of this test is to demonstrate the results which are obtained in less idealised conditions and briefly examine the usefulness to trajectory optimisation. It is evident from Figures 10 to 14 that time domain presentation and analysis of 6-DOF actuator power consumption is not convenient. Removing the time element and plotting the actuator load force against the speed give a single snapshot summary of a whole trajectory.

The aileron force-velocity plot for the turbulent mission is shown in Figure 20, where the mean value shows all motion rotates around zero velocity. This is the expected result since control surface motion occurs around a central zero location, but on the Aerosonde it has to maintain a constant force to oppose the rolling torque applied by the single propeller. It should be observed that the mean exists on the border between a motoring and generating quadrant, implying zero energy transfer. This is correct because the figure presents the mechanical energy delivered; the electrical energy plot would depend on the power converter used to drive the actuator. If no regeneration energy is stored (by using a dissipating resistor) the energy in the generating quadrants is wasted; the mean will move into the motoring quadrant and equal the mean power used in the current trajectory. Using the force-velocity plot (or indeed voltage-current) technique, a set of

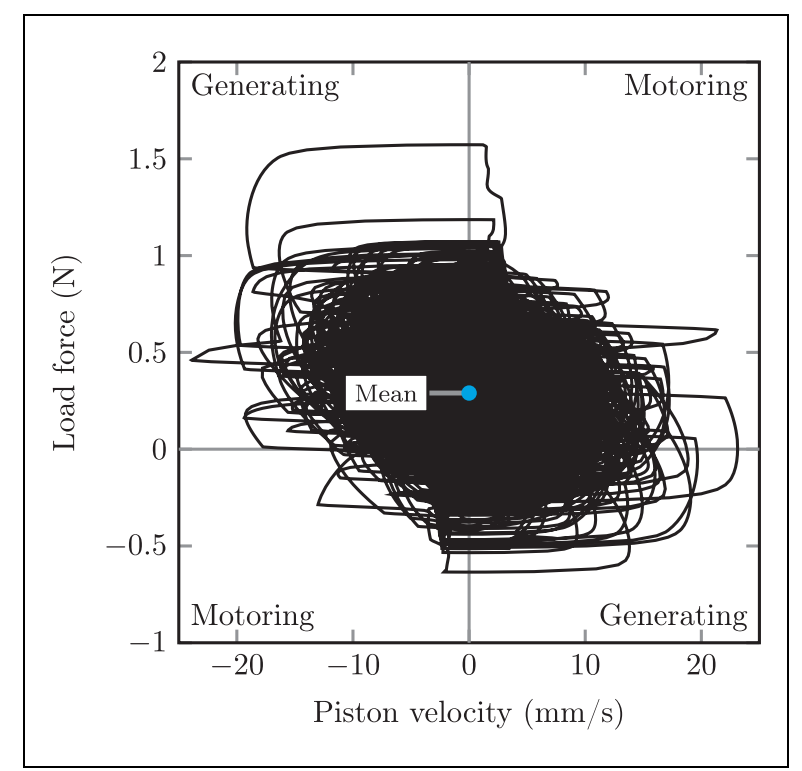

Figure 20. Force-speed plot of the starboard aileron actuator following an arbitrary trajectory in turbulence.

manoeuvres could be performed and used to populate lookup tables that are used for steady state approximations of dynamic manoeuvres.

One observation from the turbulent test is that the simulation speed decreases significantly; since there are no steady state sections of the flight, the solver time step is unable to increase at any point. One point to note is that if a trajectory optimisation task in windy conditions is sought, the noise sources must be pseudo-random and be identical for each separate trajectory execution.

\section{Conclusion}

A simulation tool has been successfully developed to investigate dynamic actuator power consumption during trajectory following manoeuvres. The tool is designed for high-speed execution by providing top level electrical power consumption data without modelling detailed electrical effects such as harmonics of the servo drive signals.

The paper demonstrates the application of the model to trajectory optimisation processes, by creating a simple set of manoeuvres to which the optimum is obvious; one banked turn to a new heading at various turn rates. These trajectories are processed by the model and a value of average motor electrical power is returned. The result produced by the model is that low turn rates require less energy than high turn rates (12.8 times less for the $90^{\circ}$ turn, neglecting the servocontroller losses), due to the reduced aerodynamic loads and lower excitement of cross coupled axes of motion. When including a constant loss approximation for the servocontrollers, the reduction decreases significantly to a factor of 1.001 .

While the actuator models may not be true representations of their nonlinear real world counterparts, 
when applied to trajectory optimisation they still provide useful relative estimations for aircraft component sizing and operation when compared against themselves. That is, comparing one possible trajectory against another.

The main complication when using this technique to analyse power consumption of actuators is the high sensitivity to the FCS and guidance algorithm used. In some instances this may be considered a benefit if the focus of the work is to analyse energy efficient FCSs such as comparing TECS/THCS to classic single input, single output autopilots. The presented methodology is capable of doing this analysis, but time must be spent to implement and tune each controller to be assessed. This includes balancing the trade-off between high computational performance and control performance - fast control means high frequencies and thus small timesteps.

The structure of the EMA actuator model is found to be suitable for the task of low frequency dynamic power consumption simulation. The greatest weakness is currently the friction model, which provides zero friction torque at zero velocity. Since the actuators are most often stationary throughout a flight, this omission causes a significant underestimation of the power consumption.

The aerodynamic load estimator available for use in this paper does not produce valid results for low Reynolds number wings. Although the trends in this paper are correct, the numerical validity of the simulation tool would be improved with more detailed aerodynamic load estimation methods.

Recommended future work involves improving the guidance algorithm to take into account the TTB for the aircraft in a robust manner. The results produced in this work are skewed due to suboptimal path following, the associated errors in distance travelled could be reduced by an improved guidance controller.

Other recommended additions are to include an engine model which simulates the change in fuel burn due to power off-takes, coupled with an electrical generator model. This would allow a direct affect on fuel burn due to electrical system demands to be quantitatively analysed.

Future addition of an experimentally validated actuator model which includes presliding and breakaway characteristics would significantly improve the numerical accuracy of the method. Another key addition would be the inclusion of power electronic converter losses which are required to drive the actuators.

\section{Declaration of Conflicting Interests}

The author(s) declared no potential conflicts of interest with respect to the research, authorship, and/or publication of this article.

\section{Funding}

The author(s) received no financial support for the research, authorship, and/or publication of this article.

\section{References}

1. Cooper MA, Lawson CP, Quaglia D, et al. Towards trajectory prediction and optimisation for energy efficiency of an aircraft with electrical and hydraulic actuation systems. In: 28th international congress of the aeronautical sciences, Brisbane, Australia, 23-28 September 2012.

2. Du X, Dixon R, Goodall RM, et al. Modelling and control of a high redundancy actuator. Mechatronics 2010; 20: 102-112.

3. Olaru D, Puiu GC, Balan LC, et al. A new model to estimate friction torque in a ball screw system. Product engineering. Netherlands: Springer, 2005, pp.333-346.

4. Portescap, 17S78 brush DC coreless motor. Available at: http://www.portescap.com/products/brush-dcmotor/17s78-brush-dc-coreless-motor (accessed 8 April 2017).

5. Roskam J. Airplane design. vol. VI. Ottawa, Kansas: Roskam Aviation \& Engineering Corporation, 1987.

6. Scholz D. Equations for a preliminary actuator design. Deutsche Airbus, TN-EV52-360/91, Germany, August 1991.

7. McGeer T and Vagners J. Flying the Atlantic - without a pilot. GPS World February 1999.

8. Beard R and McLain T. Small unmanned aircraft: theory and practice. Princeton, $\mathrm{NJ}$ : Princeton University Press, 2012.

9. Lambregts AA. Automatic flight controls. Federal Aviation Administration, 1998. Available at: https:// faaco.faa.gov/attachments/KNVLPAP6.pdf (accessed 2 January 2013).

10. Lambregts AA. Generalized automatic and augmented manual flight control. Berlin Technical University Colloquium May 2006; Available at: http://www.fmra. tu-berlin.de/fileadmin/fg162/Dokumente/Downloads/ Automatic_and_Augmented_Manual_FC.pdf (accessed 2 January 2013).

11. Chudy P and Rzucidlo P. TECS/THCS based flight control system for general aviation. In: AIAA, modeling and simulation technologies conference, Chicago, IL, 10 August 2009.

12. Bruce KR, Kelly JR and Person LH. NASA B737 flight test results of the total energy control system, NASA CR-178285, January 1987.

13. Bruzzini MA. Development of a TECS control law for the lateral directional axis of the McDonnell Douglas F15 Eagle. PhD Thesis, University of Washington, December 1994.

14. Bruce KR. Integrated autopilot/autothrottle for the NASA TSRV B-737 aircraft: design and verification by nonlinear simulation. NASA CR-4217, 1989.

15. Faleiro L and Lambregts AA. Analysis and tuning of a 'total energy control system' control law using eigenstructure assignment. Aerospace Sci Technol 1999; 3: $127-140$. 


\section{Appendix}

\section{Notation}

\begin{tabular}{|c|c|c|}
\hline$A_{w}$ & control surface area & $\mathrm{m}^{2}$ \\
\hline$B_{v}$ & actuator viscous friction & $\mathrm{Nm} / \mathrm{rad} \mathrm{s}^{-1}$ \\
\hline $\bar{c}$ & $\begin{array}{l}\text { control surface mean aerody- } \\
\text { namic chord }\end{array}$ & $\mathrm{m}$ \\
\hline$C_{h}$ & hinge moment coefficient & - \\
\hline$C_{h \alpha}$ & $\begin{array}{l}\text { hinge moment angle of attack } \\
\text { derivative }\end{array}$ & - \\
\hline$C_{h \delta}$ & $\begin{array}{l}\text { hinge moment control surface } \\
\text { angle derivative }\end{array}$ & - \\
\hline $\boldsymbol{F}$ & sum of forces on aircraft & $\mathrm{N}$ \\
\hline$F_{1}$ & actuator load force & $\mathrm{N}$ \\
\hline $\boldsymbol{H}_{b}$ & $\begin{array}{l}\text { angular momentum of the } \\
\text { aircraft in the body frame }\end{array}$ & $\mathrm{kg} \mathrm{m}^{2} \mathrm{~s}^{-1}$ \\
\hline J & aircraft moment of inertia & $\mathrm{kg} \mathrm{m}^{2}$ \\
\hline
\end{tabular}

\begin{tabular}{|c|c|c|}
\hline$K_{m}$ & actuator screw stiffness & $\mathrm{N} / \mathrm{m}$ \\
\hline$m$ & aircraft mass & $\mathrm{Kg}$ \\
\hline$M$ & sum of moments on aircraft & $\mathrm{Nm}$ \\
\hline M & control surface equivalent mass & $\mathrm{Kg}$ \\
\hline$p$ & actuator gear and screw ratio & - \\
\hline$q$ & dynamic pressure & $\mathrm{Pa}$ \\
\hline$T_{H M}$ & aerodynamic hinge moment & $\mathrm{Nm}$ \\
\hline$T_{m}$ & motor load torque & $\mathrm{Nm}$ \\
\hline$v_{b}$ & $\begin{array}{l}{\left[\begin{array}{ll}u & v\end{array}\right]^{T} \text {, velocity with regards to }} \\
\text { the inertial frame, expressed } \\
\text { in the body frame }\end{array}$ & $\mathrm{ms}^{-1}$ \\
\hline$x_{l}$ & actuator load linear position & $\mathrm{m}$ \\
\hline$x_{m}$ & $\begin{array}{l}\text { actuator ball screw nut linear } \\
\text { position }\end{array}$ & $\mathrm{m}$ \\
\hline$\alpha$ & angle of attack & $\mathrm{rad}$ \\
\hline$\delta$ & control surface deflection angle & $\mathrm{rad}$ \\
\hline$\theta_{m}$ & motor shaft angle & rad \\
\hline$\omega_{b}$ & {$\left[\begin{array}{lll}p & q & r\end{array}\right]^{T}$, body axis angular rates } & $\operatorname{rad~s}^{-1}$ \\
\hline
\end{tabular}


2017-06-13

\section{Simulating actuator energy consumption for trajectory optimisation}

Cooper, Michael

SAGE

Cooper M, Lawson C, Shahneh AZ. (2018) Simulating actuator energy consumption for trajectory optimisation. Proceedings of the Institution of Mechanical Engineers, Part G: Journal of Aerospace Engineering, Volume 232, Issue 11, September 2018, pp. 2178-2192 http://dx.doi.org/10.1177/0954410017710271

Downloaded from Cranfield Library Services E-Repository 